\title{
RADIO AND $\gamma$-RAY CONSTRAINTS ON THE EMISSION GEOMETRY AND BIRTHPLACE OF PSR J2043+2740
}

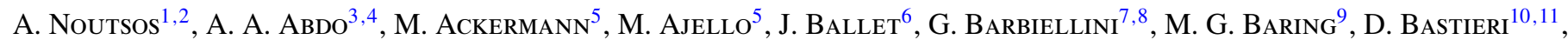
K. Bechtol ${ }^{5}$, R. Bellazzini ${ }^{12}$, B. Berenji ${ }^{5}$, E. Bonamente ${ }^{13,14}$, A. W. Borgland ${ }^{5}$, J. BregeON ${ }^{12}$, A. BREZ $^{12}$, M. BRigidA $^{15,16}$, P. Bruel ${ }^{17}$, R. Buehler ${ }^{5}$, G. Busetto ${ }^{10,11}$, G. A. Caliandro ${ }^{18}$, R. A. Cameron ${ }^{5}$, F. Camilo ${ }^{19}$, P. A. Caraveo $^{20}$,

J. M. Casandian ${ }^{6}$, C. Cecchi ${ }^{13,14}$, Ö. Çelik ${ }^{21,22,23}$, S. Chaty ${ }^{6}$, A. Chekhtman ${ }^{3,24}$, J. Chiang $^{5}$, S. Ciprini ${ }^{14}$, R. Claus ${ }^{5}$, I. Cognard ${ }^{25}$, J. Cohen-Tanugi ${ }^{26}$, S. Colafrancesco $^{27}$, S. Cutini $^{27}$, C. D. Dermer ${ }^{3}$, F. De Palma $^{15,16}$, P. S. Drell ${ }^{5}$, D. Dumora ${ }^{28}$, C. M. EA ${ }^{2}$, C. Favuzzi ${ }^{15,16}$, E. C. Ferrara ${ }^{21}$, W. B. Focke ${ }^{5}$, M. Frailis ${ }^{29,30}$, P. C. C. Freire ${ }^{1}$, Y. Fukazawa ${ }^{31}$, S. FunK ${ }^{5}$, P. Fusco ${ }^{15,16}$, F. Gargano ${ }^{16}$, S. Germani ${ }^{13,14}$, N. Giglietto ${ }^{15}, 16$, F. Giordano ${ }^{15}$, , $^{6}$, M. Giroletti $^{32}$, G. Godfrey $^{5}$, P. Grandi ${ }^{33}$, I. A. Grenier ${ }^{6}$, J. E. Grove ${ }^{3}$, L. Guillemot ${ }^{1}$, S. Guiriec ${ }^{34}$, A. K. Harding ${ }^{21}$, R. E. Hughes ${ }^{35}$, M. S. Jackson ${ }^{36,37}$, G. Jóhannesson ${ }^{5}$, A. S. Johnson ${ }^{5}$, T. J. Johnson ${ }^{21,38}$, W. N. Johnson ${ }^{3}$, S. Johnston ${ }^{39}$, T. KAMAE ${ }^{5}$, H. KATAGIRI ${ }^{31}$,

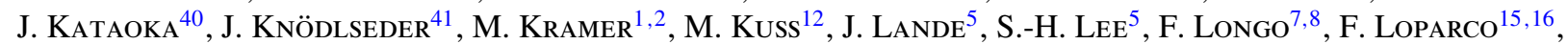
M. N. Lovellette ${ }^{3}$, P. Lubrano ${ }^{13,14}$, A. G. Lyne ${ }^{2}$, A. MaKeEv ${ }^{3,24}$, M. Marelli ${ }^{20}$, M. N. MAZZIOTtA ${ }^{16}$, J. E. McEneRY $^{21,38}$, J. Mehault ${ }^{26}$, P. F. Michelson ${ }^{5}$, T. Mizuno ${ }^{31}$, C. Monte ${ }^{15}, 16$, M. E. Monzani ${ }^{5}$, A. Morselli ${ }^{42}$, I. V. Moskalenko $^{5}$, S. Murgia ${ }^{5}$, M. Naumann-Godo ${ }^{6}$, P. L. Nolan ${ }^{5}$, E. Nuss ${ }^{26}$, T. Ohsugi ${ }^{43}$, A. Okumura ${ }^{44}$, N. Omodei $^{5}$, E. Orlando $^{45}$, J. F. Ormes ${ }^{46}$, J. H. Panetta ${ }^{5}$, D. Parent ${ }^{3,24}$, V. Pelassa ${ }^{26}$, M. Pepe ${ }^{13,14}$, M. Persic ${ }^{7,30}$, M. Pesce-Rollins ${ }^{12}$, F. Piron ${ }^{26}$, T. A. Porter ${ }^{5}$, S. Rainò ${ }^{15,16}$, P. S. RaY ${ }^{3}$, M. Razzano ${ }^{12}$, A. Reimer ${ }^{5,47}$, O. Reimer ${ }^{5,47}$, T. RePoseur ${ }^{28}$, R. W. Romani $^{5}$, H. F.-W. SAdrozinski ${ }^{48}$, A. SAnder ${ }^{35}$, P. M. Saz Parkinson ${ }^{48}$, C. Sgrò ${ }^{12}$, E. J. Siskind ${ }^{49}$, D. A. SMith ${ }^{28}$, P. D. SMith $^{35}$, G. Spandre ${ }^{12}$, P. Spinelli ${ }^{15,16}$, B. W. Stappers ${ }^{2}$, M. S. Strickman ${ }^{3}$, D. J. Suson ${ }^{50}$, H. TAKahashi $^{43}$, T. TAnaka $^{5}$, G. Theureau ${ }^{25}$, D. J. Thompson ${ }^{21}$, S. E. Thorsett ${ }^{48}$, O. Tibolla ${ }^{51}$, D. F. Torres ${ }^{18,52}$, A. Tramacere ${ }^{5,53,54}$, T. L. Usher ${ }^{5}$, J. Vandenbroucke ${ }^{5}$, G. Vianello ${ }^{5,53}$, N. Vilchez ${ }^{41}$, M. Villata ${ }^{55}$, V. Vitale ${ }^{42,56}$, A. von Kienlin ${ }^{45}$, A. P. Waite ${ }^{5}$, P. Wang ${ }^{5}$, K. Watters ${ }^{5}$, P. Weltevrede ${ }^{2}$, B. L. Winer ${ }^{35}$, K. S. Wood ${ }^{3}$, ANd M. ZiegleR ${ }^{48}$

${ }^{1}$ Max-Planck-Institut für Radioastronomie, Auf dem Hügel 69, 53121 Bonn, Germany; anoutsos@ mpifr-bonn.mpg.de, guillemo@mpifr-bonn.mpg.de

${ }^{2}$ Jodrell Bank Centre for Astrophysics, School of Physics and Astronomy, The University of Manchester, M13 9PL, UK

${ }^{3}$ Space Science Division, Naval Research Laboratory, Washington, DC 20375, USA

${ }^{4}$ National Research Council Research Associate, National Academy of Sciences, Washington, DC 20001, USA

${ }^{5}$ W. W. Hansen Experimental Physics Laboratory, Kavli Institute for Particle Astrophysics and Cosmology, Department of Physics and SLAC National Accelerator Laboratory, Stanford University, Stanford, CA 94305, USA

${ }^{6}$ Laboratoire AIM, CEA-IRFU/CNRS/Université Paris Diderot, Service d'Astrophysique, CEA Saclay, 91191 Gif sur Yvette, France

${ }^{7}$ Istituto Nazionale di Fisica Nucleare, Sezione di Trieste, I-34127 Trieste, Italy ${ }^{8}$ Dipartimento di Fisica, Università di Trieste, I-34127 Trieste, Italy

${ }^{9}$ Department of Physics and Astronomy, Rice University, MS-108, P.O. Box 1892, Houston, TX 77251, USA

${ }^{10}$ Istituto Nazionale di Fisica Nucleare, Sezione di Padova, I-35131 Padova, Italy

${ }^{11}$ Dipartimento di Fisica “G. Galilei,” Università di Padova, I-35131 Padova, Italy ${ }^{12}$ Istituto Nazionale di Fisica Nucleare, Sezione di Pisa, I-56127 Pisa, Italy

${ }^{13}$ Istituto Nazionale di Fisica Nucleare, Sezione di Perugia, I-06123 Perugia, Italy

${ }^{14}$ Dipartimento di Fisica, Università degli Studi di Perugia, I-06123 Perugia, Italy

${ }^{15}$ Dipartimento di Fisica "M. Merlin" dell'Università e del Politecnico di Bari, I-70126 Bari, Italy

${ }^{16}$ Istituto Nazionale di Fisica Nucleare, Sezione di Bari, 70126 Bari, Italy

${ }^{17}$ Laboratoire Leprince-Ringuet, École polytechnique, CNRS/IN2P3, Palaiseau, France

${ }^{18}$ Institut de Ciencies de l'Espai (IEEC-CSIC), Campus UAB, 08193 Barcelona, Spain

${ }^{19}$ Columbia Astrophysics Laboratory, Columbia University, New York, NY 10027, USA

${ }^{20}$ INAF-Istituto di Astrofisica Spaziale e Fisica Cosmica, I-20133 Milano, Italy ${ }^{21}$ NASA Goddard Space Flight Center, Greenbelt, MD 20771, USA

${ }^{22}$ Center for Research and Exploration in Space Science and Technology (CRESST) and NASA Goddard Space Flight Center, Greenbelt, MD 20771, USA

${ }^{23}$ Department of Physics and Center for Space Sciences and Technology, University of Maryland Baltimore County, Baltimore, MD 21250, USA ${ }^{24}$ George Mason University, Fairfax, VA 22030, USA

${ }^{25}$ Laboratoire de Physique et Chemie de l'Environnement, LPCE UMR 6115 CNRS, F-45071 Orléans Cedex 02, and Station de radioastronomie de Nançay, Observatoire de Paris, CNRS/INSU, F-18330 Nançay, France

${ }^{26}$ Laboratoire de Physique Théorique et Astroparticules, Université Montpellier 2, CNRS/IN2P3, Montpellier, France

${ }^{27}$ Agenzia Spaziale Italiana (ASI) Science Data Center, I-00044 Frascati (Roma), Italy

${ }^{28}$ Université Bordeaux 1, CNRS/IN2p3, Centre d'Études Nucléaires de Bordeaux Gradignan, 33175 Gradignan, France

${ }^{29}$ Dipartimento di Fisica, Università di Udine and Istituto Nazionale di Fisica Nucleare, Sezione di Trieste, Gruppo Collegato di Udine, I-33100 Udine, Italy

${ }^{30}$ Osservatorio Astronomico di Trieste, Istituto Nazionale di Astrofisica, I-34143 Trieste, Italy

${ }^{31}$ Department of Physical Sciences, Hiroshima University, Higashi-Hiroshima, Hiroshima 739-8526, Japan

${ }^{32}$ INAF Istituto di Radioastronomia, 40129 Bologna, Italy

${ }^{33}$ INAF-IASF Bologna, 40129 Bologna, Italy

${ }^{34}$ Center for Space Plasma and Aeronomic Research (CSPAR), University of Alabama in Huntsville, Huntsville, AL 35899, USA

${ }^{35}$ Department of Physics, Center for Cosmology and Astro-Particle Physics, The Ohio State University, Columbus, OH 43210, USA

${ }^{36}$ Department of Physics, Royal Institute of Technology (KTH), AlbaNova, SE-106 91 Stockholm, Sweden

${ }^{37}$ The Oskar Klein Centre for Cosmoparticle Physics, AlbaNova, SE-106 91 Stockholm, Sweden

${ }^{38}$ Department of Physics and Department of Astronomy, University of Maryland, College Park, MD 20742, USA

${ }^{39}$ Australia Telescope National Facility, CSIRO, Epping, NSW 1710, Australia

${ }^{40}$ Research Institute for Science and Engineering, Waseda University, 3-4-1 Okubo, Shinjuku, Tokyo 169-8555, Japan

${ }^{41}$ Centre d'Étude Spatiale des Rayonnements, CNRS/UPS, BP 44346, F-30128 Toulouse Cedex 4, France

42 Istituto Nazionale di Fisica Nucleare, Sezione di Roma “Tor Vergata," I-00133 Roma, Italy

${ }^{43}$ Hiroshima Astrophysical Science Center, Hiroshima University, Higashi-Hiroshima, Hiroshima 739-8526, Japan

${ }^{44}$ Institute of Space and Astronautical Science, JAXA, 3-1-1 Yoshinodai, Sagamihara, Kanagawa 229-8510, Japan 


\author{
${ }^{45}$ Max-Planck Institut für extraterrestrische Physik, 85748 Garching, Germany \\ ${ }^{46}$ Department of Physics and Astronomy, University of Denver, Denver, CO 80208, USA \\ ${ }^{47}$ Institut für Astro- und Teilchenphysik and Institut für Theoretische Physik, Leopold-Franzens-Universität Innsbruck, A-6020 Innsbruck, Austria \\ ${ }^{48}$ Santa Cruz Institute for Particle Physics, Department of Physics and Department of Astronomy and Astrophysics, \\ University of California at Santa Cruz, Santa Cruz, CA 95064, USA \\ ${ }^{49}$ NYCB Real-Time Computing Inc., Lattingtown, NY 11560-1025, USA \\ ${ }^{50}$ Department of Chemistry and Physics, Purdue University Calumet, Hammond, IN 46323-2094, USA \\ ${ }^{51}$ Institut für Theoretische Physik and Astrophysik, Universität Würzburg, D-97074 Würzburg, Germany \\ ${ }^{52}$ Institució Catalana de Recerca i Estudis Avançats (ICREA), Barcelona, Spain \\ ${ }^{53}$ Consorzio Interuniversitario per la Fisica Spaziale (CIFS), I-10133 Torino, Italy \\ ${ }^{54}$ INTEGRAL Science Data Centre, CH-1290 Versoix, Switzerland \\ 55 INAF, Osservatorio Astronomico di Torino, I-10025 Pino Torinese (TO), Italy \\ ${ }^{56}$ Dipartimento di Fisica, Università di Roma “Tor Vergata," I-00133 Roma, Italy \\ Received 2010 September 22; accepted 2010 December 6; published 2011 January 24
}

\begin{abstract}
We report on the first year of Fermi $\gamma$-ray observations of pulsed high-energy emission from the old PSR J2043 + 2740. The study of the $\gamma$-ray efficiency of such old pulsars gives us an insight into the evolution of pulsars' ability to emit in $\gamma$ rays as they age. The $\gamma$-ray light curve of this pulsar above $0.1 \mathrm{GeV}$ is clearly defined by two sharp peaks, $0.353 \pm 0.035$ periods apart. We have combined the $\gamma$-ray profile characteristics of PSR J2043 + 2740 with the geometrical properties of the pulsar's radio emission, derived from radio-polarization data, and constrained the pulsar-beam geometry in the framework of a two-pole caustic (TPC) and an outer gap (OG) model. The ranges of magnetic inclination and viewing angle were determined to be $\{\alpha, \zeta\} \sim\left\{52^{\circ}-57^{\circ}, 61^{\circ}-68^{\circ}\right\}$ for the TPC model, and $\{\alpha, \zeta\} \sim\left\{62^{\circ}-73^{\circ}, 74^{\circ}-81^{\circ}\right\}$ and $\{\alpha, \zeta\} \sim\left\{72^{\circ}-83^{\circ}, 60^{\circ}-75^{\circ}\right\}$ for the OG model. Based on this geometry, we assess possible birth locations for this pulsar and derive a likely proper motion, sufficiently high to be measurable with VLBI. At a characteristic age of 1.2 Myr, PSR J2043 + 2740 is the third oldest of all discovered, non-recycled, $\gamma$-ray pulsars: it is twice as old as the next oldest, PSR J0357 + 32, and younger only than the recently discovered PSR J1836 + 5925 and PSR J2055 + 25, both of which are at least five and ten times less energetic, respectively.
\end{abstract}

Key words: gamma rays: stars - pulsars: individual (PSR J2043 + 2740)

Online-only material: color figures

\section{INTRODUCTION}

Before the advent of the Fermi $\gamma$-ray Space Telescope (hereafter Fermi), the Compton Gamma Ray Observatory (CGRO) succeeded in detecting $\mathrm{GeV}$ emission from a handful of pulsars, while a much higher number of $\gamma$-ray sources remained unidentified (Hartman et al. 1999). It is generally expected that the most energetic pulsars, i.e., with spin-down luminosity $L_{\mathrm{sd}}=4 \pi^{2} I \dot{P} / P^{3}>10^{34} \mathrm{erg} \mathrm{s}^{-1}\left(I=10^{45} \mathrm{~g} \mathrm{~cm}^{2}\right.$ is the neutron star's moment of inertia, $P$ is the spin period, and $\dot{P}$ its first derivative), are the best candidates for detectable $\gamma$-ray emission (Thompson et al. 1999). They are typically young pulsars of characteristic spin-down age $\tau_{\mathrm{c}}=P /(2 \dot{P})<100 \mathrm{kyr}$, with $\dot{P}>10^{-15}$ and $P \lesssim 0.1 \mathrm{~s}$. Those expectations received support with the discovery of the six EGRET pulsars, all of which have $L_{\text {sd }}>3 \times 10^{34} \mathrm{erg} \mathrm{s}^{-1}$. In that sample, the two oldest pulsars were PSR B1055 - 52, with a spin-down age of $\tau_{\mathrm{c}}=535 \mathrm{kyr}$, and the radio-quiet Geminga, with $\tau_{\mathrm{c}}=342 \mathrm{kyr}$; both these pulsars are considered middle-aged amongst the known sample of non-recycled pulsars (e.g., Figure 2 in Abdo et al. 2010a).

The Fermi satellite was successfully launched on 2008 June 11. During the first six months of the mission, data collected with the Large Area Telescope (LAT) - the main instrument on board Fermi-were analysed for pulsed $\gamma$ rays from a preselected list of energetic radio pulsars (Smith et al. 2008). The list of pulsars was selected based on the spin-down luminosity, so that $L_{\mathrm{sd}}>10^{34} \mathrm{erg} \mathrm{s}^{-1}$. Not surprisingly, the vast majority of non-recycled pulsars on the candidate list have $\tau_{\mathrm{c}}<10^{3} \mathrm{kyr}$. One of the few exceptions is the $96 \mathrm{~ms}$ pulsar PSR J2043 + 2740 $\left(P=0.0961 \mathrm{~s}, P=1.23 \times 10^{-15}\right)$, which is much older than the rest in the sample, with $\tau_{\mathrm{c}}=1.2 \times 10^{3} \mathrm{kyr}$. A recent effort to detect a high-energy signal from this pulsar was made with the AGILE space telescope by Pellizzoni et al. (2009). They reported a pulsed signature above $50 \mathrm{MeV}$, at the level of $4.2 \sigma$ above the background. However, a detection was not claimed and the authors calculated a $2 \sigma \gamma$-ray flux upper limit of $F(>100 \mathrm{MeV})<6 \times 10^{-8} \mathrm{~cm}^{-2} \mathrm{~s}^{-1}$. In addition to the flux estimation, the authors placed an upper limit on the $\gamma$-ray efficiency: $\eta=L_{\gamma} / L_{\mathrm{sd}}<0.01$, under the assumption of a $1 \mathrm{sr}$ beam and a spectral index of 2.0. Following the AGILE observations, Abdo et al. (2010a) folded the first six months of Fermi data, from the direction of PSR J2043 + 2740, with the radio-timing ephemeris supplied by Jodrell Bank (Hobbs et al. 2004). That analysis yielded the first confident detection (at nearly $5 \sigma$ ) of $\gamma$-ray pulsations from PSR J2043 +2740 .

PSR J2043 + 2740 was discovered in radio, in the Arecibo millisecond-pulsar survey at $430 \mathrm{MHz}$ (Thorsett et al. 1994). Based on its dispersion measure, $\mathrm{DM}=21.0 \pm 0.1 \mathrm{pc} \mathrm{cm}^{-3}$ (Ray et al. 1996), and the NE2001 free-electron density model of Cordes \& Lazio (2002), the distance estimate for this pulsar is $D \approx 1.8 \mathrm{kpc}$ (Manchester et al. 2005). PSR J2043 + 2740 lies near the southwestern shell of the Cygnus Loop $(\sim 15$ pc outside the observable edge), perhaps suggesting an association with the remnant. However, the evidence so far suggests that such an association is unlikely: the distance to the Cygnus Loop has been estimated to $540_{-80}^{+100} \mathrm{pc}$ (Blair et al. 2005, 2009); in addition, assuming that the pulsar was born within the observable limits of the remnant, the age of the latter ( $<12 \mathrm{kyr}$; Sankrit \& Blair 2002) suggests a transverse velocity of $>980 \mathrm{~km} \mathrm{~s}^{-1}$ for the pulsar, which is significantly higher than the average birth velocity of the known pulsar sample (400 $\pm 40 \mathrm{~km} \mathrm{~s}^{-1}$; Hobbs et al. 2005). 
Last but not least, the pulsar's characteristic age, as calculated from its spin parameters, is two orders of magnitude higher than the remnant's. Therefore, these discrepancies need to be reconciled before an association can be claimed.

There are indeed examples of age discrepancy between pulsars and their associated remnants, like in the case of PSR J0538 + 2817 and the supernova remnant (SNR) S147 (Anderson et al. 1996). This pulsar has a characteristic spindown age of $620 \mathrm{kyr}$ but has been confidently associated with the $40 \mathrm{kyr}$ remnant via pulsar timing and very long baseline interferometry (VLBI; Romani \& Ng 2003; Kramer et al. 2003; $\mathrm{Ng}$ et al. 2007). Those VLBI measurements, combined with previous X-ray observations of the pulsar's thermal profile (McGowan et al. 2003), have revealed a hot neutron star with a transverse velocity of $400 \mathrm{~km} \mathrm{~s}^{-1}$, which matches the observed average. The above studies concluded that PSR J0538 + 2817 must have been born with a slow initial spin period, very close to that observed today, thus invalidating the usual assumption of a short birth period for this pulsar.

The properties of PSR J2043 + 2740 make it an intriguing pulsar for high-energy studies with Fermi: it is one of the shortest-period, non-recycled $\gamma$-ray pulsars without a known SNR association. Indeed, there are a number of recent Fermi detections of non-recycled pulsars that have shorter periods than PSR J2043 + 2740 and for which there is yet no association with a remnant: i.e., PSRs J1028 - 5819, J1718 - 3825, $\mathrm{J} 1420$ - 6048, and J1813 - 1246 (Abdo et al. 2010a). However, these pulsars are $\sim 10^{2}$ times younger than PSR J2043 + 2740 and are much more likely to be associated with a nearby remnant; on the contrary, if the characteristic age for PSR J2043 + 2740 corresponds to its true age (see Section 4.2), then it is very unlikely that there would be any visible remnant left for an association to be possible. Moreover, the fast rotation of PSR J2043 + 2740 implies a relatively high spin-down luminosity $\left(L_{\mathrm{sd}}=5.6 \times 10^{34} \mathrm{erg} \mathrm{s}^{-1}\right)$ compared to pulsars of similar characteristic age. This means that, in terms of energetics, PSR J2043 + 2740 is on a par with much younger pulsars, like PSR J1835 - 0643, which is an order of magnitude younger.

Apart from arousing observational interest, this pulsar's physical properties are also attractive in theoretical investigations. In terms of $\gamma$-ray observability, $L_{\gamma} / D^{2}$, PSR J2043 +2740 has been previously considered as a strong candidate for $\gamma$ ray emission, in the framework of both polar cap (PC) and outer gap (OG) models (Rudak \& Dyks 1998; Hibschman 2002; Cheng \& Zhang 1998; McLaughlin \& Cordes 2000). It should be noted, however, that the majority of Fermi pulsar observations to date have produced $\gamma$-ray spectra that disagree with the predicted super-exponential cutoffs of PC models (e.g., Abdo et al. 2009, 2010b). Nevertheless, even within the framework of outer-magnetospheric models, there exist alternative emission geometries to the traditional OG that describe the production of $\gamma$ rays at high altitudes above the pulsar surface: one such geometry is that of the two-pole caustic (TPC) model (Dyks \& Rudak 2003). The detection of PSR J2043 + 2740 makes it possible to test the predictions of the above models for this pulsar against the properties derived from observations. In Section 4.1, we discuss the results from the derived emission geometry from radio-polarization data and the $\gamma$-ray light curves, and what those suggest for the model describing this pulsar's emission.

Furthermore, the measurement of the $\gamma$-ray efficiency, $\eta=$ $L_{\gamma} / L_{\text {sd }}$, for the old PSR J2043 +2740 extends the studies of $\eta$ by a factor of two in characteristic age. This can help us confirm or reject previous claims for an increasing $\gamma$-ray efficiency with pulsar age (Buccheri 1980; Harding 1981; Zhang $\&$ Cheng 1998). A discussion on this subject can be found in Section 4.2 .

The present article reports on the results of our analysis of 14 months of Fermi data from the direction of PSR J2043 + 2740. The LAT instrument on board Fermi is sensitive to $\gamma$ rays of energies from 0.02 to $300 \mathrm{GeV}$; its sensitivity is an order of magnitude higher than EGRET and more than three times higher compared to AGILE above $0.5 \mathrm{GeV}$ (Atwood et al. 2009). Using the data collected with LAT during the first 14 months of operation (2008 August 4 to 2009 October 17), we have detected $\gamma$-ray pulsations at a very high significance $(\approx 7 \sigma)$ from PSR J2043 + 2740. This work strengthens the previously published detection of this pulsar with Fermi data (Abdo et al. 2010a).

\section{RADIO OBSERVATIONS AND DATA ANALYSIS}

The analysis of radio data from PSR J2043 + 2740 consisted of two parts: (1) the analysis of timing data from Jodrell Bank observations at $1.4 \mathrm{GHz}$, from which we derived a rotational ephemeris that was used to fold the $\gamma$-ray data; and (2) the analysis of polarimetric data from observations at $1.4 \mathrm{GHz}$ with the Effelsberg radio telescope, which we used to determine the beam geometry of the pulsar's emission.

\subsection{Timing Data and Analysis}

The rotational ephemeris for PSR J2043 + 2740, which is required to fold the $\gamma$-ray photons with the pulsar's period, was obtained from regular timing observations with the Lovell $76 \mathrm{~m}$ radio telescope, at $1.4 \mathrm{GHz}$. The Jodrell Bank radio observatory has been timing this pulsar regularly since 1996, as part of its extensive pulsar-timing program (Hobbs et al. 2004). In our analysis, we used timing data that spanned from 2008 June 17 to 2009 October 17, covering 78, 12 minute integrations of the radio pulses, from each of which a precise time of arrival (TOA) was determined. The set of TOAs was analysed with the pulsar-timing package TEMPO2 (Hobbs et al. 2006), which we used in order to fit a timing model to the TOAs, describing the pulsar rotation during the above range of dates. The only free parameters of the timing model were the pulsar spin-frequency and its first two time derivatives, while the pulsar position and DM were deemed sufficiently well-determined from previous observations. After fitting, the pulsar ephemeris was a good description of the pulsar rotation, with the rms of the timing residuals being $\approx 120 \mu$ s across the entire fitted range. The uncertainty in DM results in a possible error in the time delay between the radio and $\gamma$-ray frequencies (with $f_{\mathrm{LAT}} \rightarrow \infty$ ), via the dispersion law, $\delta t=\left(4.15 \times 10^{6} \mathrm{~ms}\right) \times \sigma_{\mathrm{DM}} / f^{2}$. Substituting for the DM uncertainty and the radio frequency gives $\delta t \approx 0.21$ $\mathrm{ms}$, which corresponds to only $0.2 \%$ phase error; this is of the same order of magnitude as the uncertainty of the ephemeris. However, given the low number of $\gamma$-ray events collected from PSR J2043 + 2740, so far, the impact of both errors on the pulsar's $\gamma$-ray light curve is negligible.

The timing parameters used in this study will be made available on the servers of the Fermi Science Support Center (FSSC). ${ }^{57}$

\subsection{Polarimetric Data and Analysis}

In addition to the timing observations, we performed polarization observations of PSR J2043 + 2740 at $1.4 \mathrm{GHz}$, with

\footnotetext{
57 http://Fermi.gsfc.nasa.gov/ssc
} 


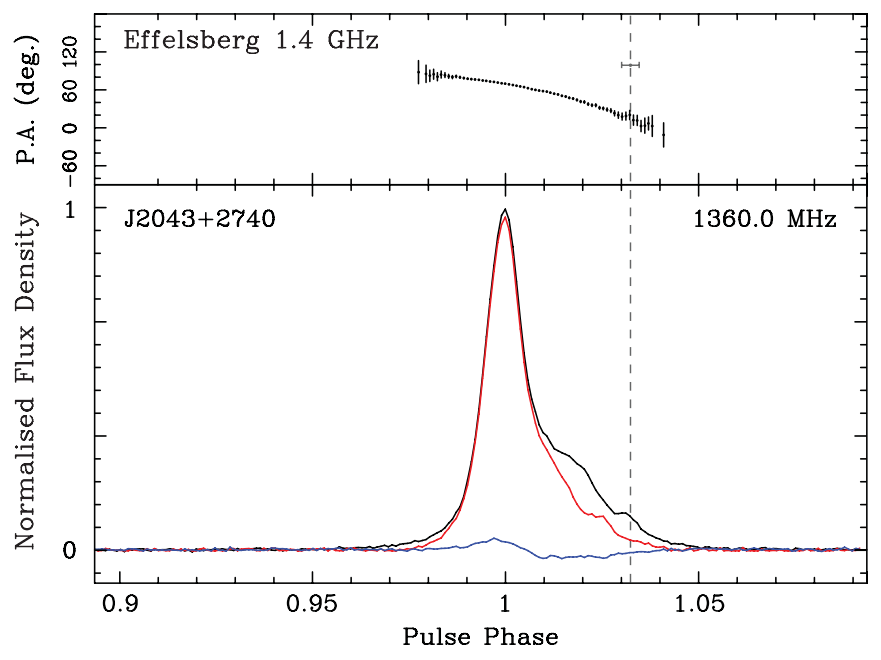

Figure 1. Polarization profile of PSR J2043 +2740, at $1.4 \mathrm{GHz}$, from a 45 minute integration with the Effelsberg radio telescope. Bottom panel: profiles of the total intensity (black, solid line), linearly polarized intensity (red line), and circularly polarized intensity (blue line). Top panel: P.A. profile (black error bars). The gray dashed line denotes the rotational phase at the minimum approach of the line of sight to the magnetic pole (i.e., $\phi_{0}$ ). The uncertainty on $\phi_{0}$ is shown with a horizontal error bar.

(A color version of this figure is available in the online journal.)

the Effelsberg $100 \mathrm{~m}$ radio telescope. The $21 \mathrm{~cm}$ receiver of the Effelsberg observatory is equipped with left and right circular polarization feeds, with a system equivalent flux density of $\approx 20 \mathrm{Jy}$. The two polarization channels were fed into a Digital Filterbank back end, which was set to de-disperse the $150 \mathrm{MHz}$ of available bandwidth, split into 1024 frequency channels. For calibration purposes, during the entire 45 minute observation we triggered the receiver's noise diode at the pulsar frequency but separated by 0.5 periods from the pulse position. The noise diode injects an artificial, pulsed signal that is $100 \%$ linearly polarized, and which can be used as a reference source for correcting for the gain differences between the polarization feeds, in the post-processing.

We used the PSRCHIVE software package (Hotan et al. 2004) to calibrate the Effelsberg data and produce a high signal-tonoise $(\mathrm{S} / \mathrm{N})$ polarization profile of PSR J2043 + 2740. The total-intensity, linear-, and circular-polarization profiles of this pulsar are shown in Figure 1. Furthermore, in the top panel of the same figure we show the polarization position angle (hereafter P.A.) profile across the pulse, given by P.A. $=0.5 \arctan (U / Q)$, where $Q$ and $U$ are the Stokes parameters of the linearly polarized emission at each pulse longitude: only P.A.s with $\mathrm{S} / \mathrm{N}>4$ are shown in the plot.

Adopting a standard rotating vector model (RVM), described in Radhakrishnan \& Cooke (1969), we determined the modelparameter space from fits to the observed P.A.s across the pulse: i.e., $\alpha$, the inclination angle between the magnetic and rotating axes; $\zeta=\alpha+\beta$, the viewing angle, where $\beta$ is the minimum angular separation between the observer's line of sight and the magnetic axis, which occurs at rotational phase $\phi_{0}$; and P.A. 0 , the P.A. at $\phi_{0}$. As is the case with many other polarized pulsars, the P.A. profile of PSR J2043 + 2740 reveals to us only part of the full swing of the magnetic axis across our field of view. Therefore, there are large uncertainties on the fitted parameters. In order to estimate the confidence intervals of $\alpha$ and $\zeta$, we fitted the P.A.s with an RVM model: we used a $500 \times 500$ grid in $\alpha=\left\{0^{\circ}-180^{\circ}\right\}$ and $\zeta=\left\{0^{\circ}-180^{\circ}\right\}$ and left $\phi_{0}$ and $\psi_{0}$ as free parameters to be fitted with a $\chi^{2}$-minimization procedure. Each grid point was then assigned to the calculated $\chi^{2}$ value from the respective fit and $1 \sigma, 3 \sigma$, and $5 \sigma$ contours were generated.

Despite the aforementioned polarization calibration procedure, which largely corrects for the parallactic angle changes during the observation and the frequency-dependent gain differences between the dipoles (for a description see Johnston 2002), the P.A.s that we measured with our back end are subjected to additional instrumental rotations by the different components between the receiver and the back end. The instrumental rotation is assumed to be time and frequency independent, and one can account and correct for this constant shift in the P.A.s by observing with the same setup a set of highly polarized pulsars with well-known polarization properties. Given that these pulsars are also calibrated, the difference between the measured P.A.s (corrected to infinite frequency) and previously published absolute P.A. values should correspond to the instrumental rotation. Application of the measured instrumental rotation to the calibrated profile of any pulsar observed with the same system should result in absolute P.A.s.

We performed polarization observations of five pulsars-PSR B0355 + 54, PSR B0740 - 28, PSR B1929 + 10, PSR B1953 + 50, and PSR B2154 + 40-with the same receiver setup and produced calibrated P.A. profiles across the pulse. Using the calibrated P.A.s, we measured the rotation measures (RMs) of these pulsars by fitting for the P.A. rotation across our $150 \mathrm{MHz}$ band. Following that, we used the resulting RMs to correct the measured P.A.s to infinite frequency. Then, we calculated the mean difference between the published values of P.A.0 and those from our measurements in our sample of five pulsars. Absolute polarization profiles for these pulsars were found in Johnston et al. (2005) and Carr (2007). The calculated $\triangle$ P.A. between the measured and published P.A.S was P.A.meas - P.A.pub $=13.6 \pm 2.8$.

For PSR J2043 + 2740, following the above procedure, we measured $\mathrm{RM}=-92.7 \pm 1.9 \mathrm{rad} \mathrm{m}^{-2}$ and corrected the P.A.s to infinite frequency, according to that value. Finally, by subtracting $\triangle$ P.A. from all measured P.A. values, we calculated the absolute polarization-angle profile shown in the top panel of Figure 1. The RVM-model contours from the radio-polarization data allowed us to place constraints on the ranges of the $\alpha$ and $\zeta$ parameters (see Section 4.1). Following the aforementioned RVM-fitting approach but, instead, using a grid in $\phi_{0}$ and P.A.0 and fitting for $\alpha$ and $\zeta$, we were able to generate confidence contours for $\phi_{0}$ and P.A. 0 (Figure 2). The best solution $\left(\chi_{\mathrm{r}}^{2} \approx\right.$ $0.8)$ gave $\phi_{0}=1.032 \pm 0.002$ and P.A. $0=17^{\circ} \pm 6^{\circ}$.

\section{3. $\gamma$-RAY OBSERVATIONS AND DATA ANALYSIS}

\subsection{Light Curve Analysis}

The raw event data from the Fermi LAT observations were subjected to cuts, in order to reduce the $\gamma$-ray background. We kept the "diffuse" class events as defined under the P6_V3 instrument response function (IRF), having the best probability of being $\gamma$-ray photons (Atwood et al 2009), and rejected all events with zenith angles greater than $105^{\circ}$ in order to reduce the contribution of the Earth's $\gamma$-ray albedo. ${ }^{58}$ We kept events within an energy-dependent radius corresponding to $68 \%$ of the point-spread function (PSF) of the LAT, from the position of $\mathrm{J} 2043+2740\left(\alpha_{\mathrm{J} 2000}=310.931, \delta_{\mathrm{J} 2000}=27.682\right)$, $\theta_{68}=0.8 \times E^{-0.8}$ with $E$ in $\mathrm{GeV}$, with a minimum value of

\footnotetext{
58 Earth's albedo of $\gamma$ rays is produced by cosmic rays interacting with the atmosphere.
} 


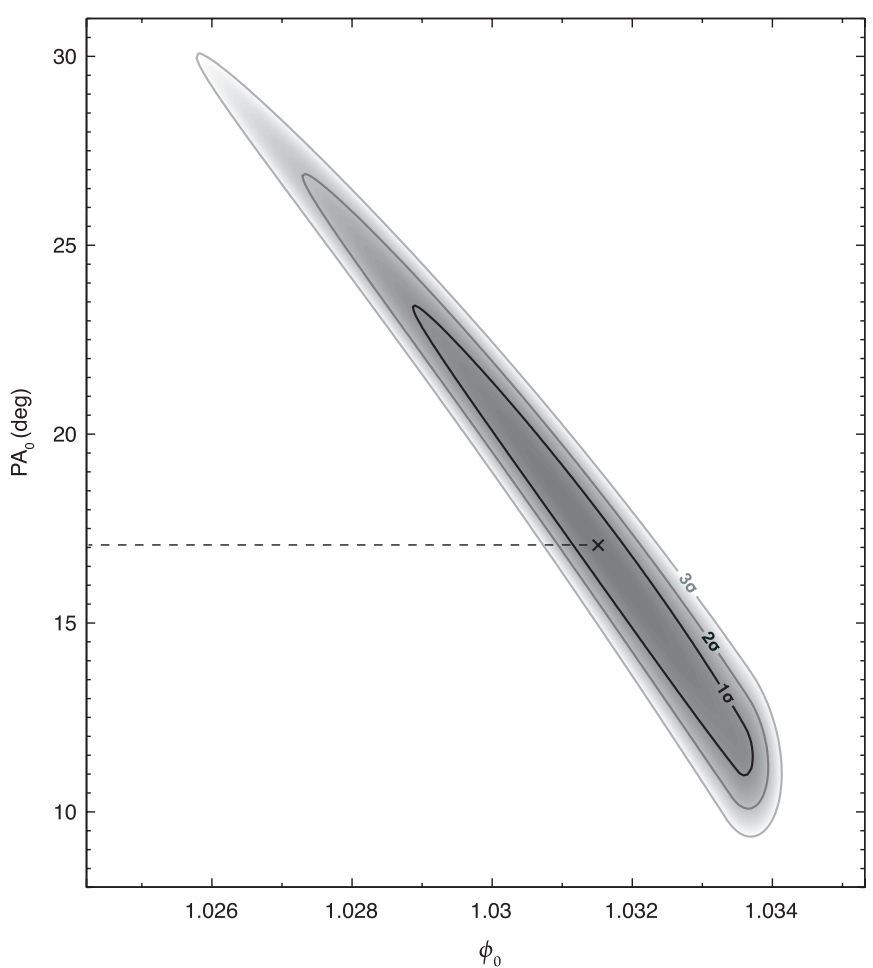

Figure 2. Confidence contours for $\phi_{0}$ and P.A.0 from fits to the polarization data of Figure 1 with an RVM model, where $\alpha$ and $\zeta$ in the model were taken from a $500 \times 500$ grid covering $0^{\circ}-180^{\circ}$ in each parameter. The best-fit pair of $\phi_{0}$ and P.A. 0 , corresponding to $\chi_{\mathrm{r}}^{2} \approx 0.8$, is shown with a cross.

0.1 for $E>10 \mathrm{GeV}$. A maximum radius of $1^{\circ}$ around the pulsar was imposed to remove as many low-energy $\gamma$ rays due to the Galactic diffuse emission as possible. The remaining 1244 photons were phase-folded using the radio ephemerides described above, and the Fermi plug-in provided by the LAT team and distributed in the TEMPO2 pulsar timing package (Hobbs et al. 2006).

Figure 3 shows the generated light curves for all events above $0.1 \mathrm{GeV}$, those between 0.1 and $0.3 \mathrm{GeV}, 0.3$ and $1 \mathrm{GeV}$, and all events above $1 \mathrm{GeV}$. The highest-energy event in the data after the application of cuts was $4.9 \mathrm{GeV}$, and falls at 0.30 in phase. The bottom plot, in the same figure, shows the radio profile of PSR J2043 + 2740 from Jodrell Bank observations at $1.4 \mathrm{GHz}$. All profiles were phase-aligned to the maximum of the radio emission (set by definition to $\phi=0$ ), to allow a comparison study between the longitude of radio and highenergy emission. The overall light curve above $0.1 \mathrm{GeV}$ shows two significant peaks of $\gamma$-ray emission, which is in agreement with previous Fermi observations (Abdo et al. 2010a), but departs from the marginally significant light curve recorded by AGILE (Pellizzoni et al. 2009). Application of the binningindependent $H$-test for uniformity (de Jager et al. 1989) to those events yielded $H=69.5$, which translates to $\mathrm{a} \approx 7 \sigma$ detection (de Jager \& Büsching 2010). Inspection of the remaining histograms showed that the phase region defining P1 in the aggregate light curve is mainly populated by $\gamma$ rays coming from the lower-energy windows, i.e., $0.1-1 \mathrm{GeV}$. In contrast, the $\gamma$ rays that form P2 in the total light curve mainly come from the highest-energy window, i.e., $>1 \mathrm{GeV}$. We therefore conclude that there is a spectral dependence of the $\gamma$-ray light curve, with the second peak harder than the first one.

In order to accurately determine the peak positions in the light curve of PSR J2043 + 2740, a C-test (de Jager 1994) was applied.

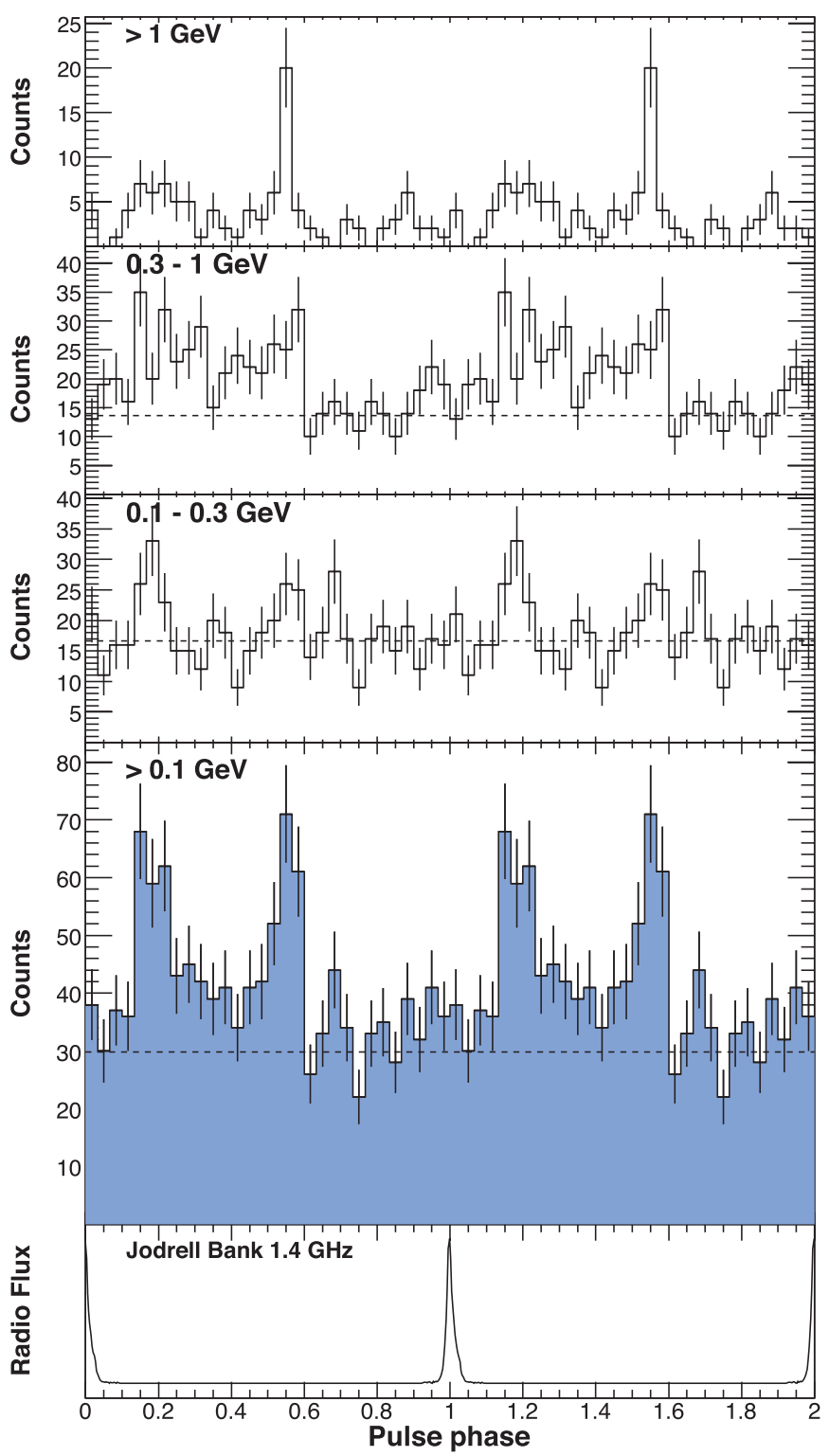

Figure 3. Top four panels: $\gamma$-ray light curves of PSR J2043 +2740 for $E>0.1 \mathrm{GeV}, 0.1 \mathrm{GeV}<E<0.3 \mathrm{GeV}, 0.3 \mathrm{GeV}<E<1 \mathrm{GeV}$, and $E>1 \mathrm{GeV}$. Bottom panel: radio profile of PSR J2043 + 2740 from Jodrell Bank observations at $1.4 \mathrm{GHz}$. The horizontal dashed lines show the background levels estimated from a surrounding annulus.

(A color version of this figure is available in the online journal.)

This test, unlike the $H$-test, is sensitive to phase and pulse-width information, which allows one to maximize the significance of the signal by scanning in phase and pulse width around the roughly known pulse positions. A grid of $200 \times 60$ trials of peak position, $\phi$, and pulse width, $W$, respectively, was generated and a probability of random occurrence was calculated for each point on the grid. The pulse width was defined as the full width at half-maximum (FWHM) of a Gaussian pulse shape. From the test, we found that $\phi_{\mathrm{P} 1}=0.20 \pm 0.03$ and $W_{\mathrm{P} 1}=0.19 \pm 0.06$, and $\phi_{\mathrm{P} 2}=0.55 \pm 0.02$ and $W_{\mathrm{P} 2}=0.06 \pm 0.01$ describe well the peak position and width.

From the above values we derive a phase lag between the maximum of the radio emission peak and the first $\gamma$-ray peak of $\delta=0.20 \pm 0.03$, and a separation between the two $\gamma$-ray peaks of $\Delta=0.35 \pm 0.04$, in agreement with previously released results (Abdo et al. 2010a). 


\subsection{Spectral Analysis}

The phase-averaged spectrum for PSR J2043 + 2740 was obtained by performing a maximum likelihood analysis (Mattox et al. 1996) of the LAT data within $15^{\circ}$ from the pulsar, using the standard tool "gtlike." The Galactic diffuse emission was modeled using the gll_iem_v02 map cube. The extragalactic diffuse and residual instrument background components were modeled jointly using the isotropic_iem_v02 template. Both models are available for download with the Fermi Science Tools. ${ }^{59}$ All sources within $15^{\circ}$ from $\mathrm{J} 2043+2740$ in the 11 month catalog of point sources were included in the analysis, and parameters for sources more than $5^{\circ}$ away from the pulsar were fixed in the fit (Abdo et al. 2010c). We modeled the emission from $\mathbf{J} 2043+2740$ as a simple power law and an exponentially cutoff power law of the following form:

$$
\frac{d N}{d E}=N_{0}\left(\frac{E}{1 \mathrm{GeV}}\right)^{-\Gamma} e^{-\frac{E}{E_{\mathrm{c}}}}
$$

In this expression, $\Gamma$ is the power-law index, $E_{\mathrm{c}}$ is the cutoff energy, and $N_{0}$ is a prefactor term. The exponentially cutoff power-law emission model is preferred at the $5.5 \sigma$ level over the simple power-law model. We obtain $N_{0}=(1.2 \pm 0.2) \times 10^{-8}$ photons $\mathrm{cm}^{-2} \mathrm{~s}^{-1} \mathrm{GeV}^{-1}, \Gamma=1.1 \pm 0.2$, and $E_{\mathrm{c}}=0.8 \pm$ $0.1 \mathrm{GeV}$. We also modeled the pulsar with a super-exponentially cutoff power law of the form $N_{0}(E / 1 \mathrm{GeV})^{-\Gamma} \exp \left[-\left(E / E_{\mathrm{c}}\right)^{\beta}\right]$, where $\beta$ was left free in the fit. We measured $\beta=1.0 \pm 0.4$, and therefore we conclude that the simple exponential cutoff power-law models the present data well.

Integrating Equation (1) for energies above $0.1 \mathrm{GeV}$ gives an integral photon flux $F_{>0.1 \mathrm{GeV}}=(2.2 \pm 0.4) \times 10^{-8}$ photons $\mathrm{cm}^{-2} \mathrm{~s}^{-1}$, and an energy flux $G_{>0.1 \mathrm{GeV}}=(1.5 \pm$ $0.2) \times 10^{-11} \mathrm{erg} \mathrm{cm}^{-2} \mathrm{~s}^{-1}$. The quoted errors are statistical only. The effect of the systematic uncertainties in the effective area on the spectral parameters is $\delta \Gamma=(+0.3,-0.1)$, $\delta E_{\mathrm{c}}=(+20 \%,-10 \%), \delta F_{>0.1 \mathrm{GeV}}=(+30 \%,-10 \%)$, and $\delta G_{>0.1 \mathrm{GeV}}=(+20 \%,-10 \%)$ (Abdo et al. 2010a). These systematic uncertainties were assessed by making the analysis as described above, but using an effective area for the LAT modified by $\pm 10 \%$ at $0.1 \mathrm{GeV}, \pm 5 \%$ at $0.5 \mathrm{GeV}$, and $\pm 20 \%$ at $10 \mathrm{GeV}$ with linear extrapolations in log space between. Our values for $\Gamma, E_{\mathrm{c}}, F_{>0.1 \mathrm{GeV}}$, and $G_{>0.1 \mathrm{GeV}}$ are in good agreement with those of Abdo et al. (2010a), but with improved statistical uncertainties.

\section{DISCUSSION}

\subsection{High-energy Models}

All recent detections by Fermi suggest that the observed $\gamma$ ray emission from pulsars is distributed over a large fraction of the celestial sphere, i.e., $f_{\Omega} \gtrsim 1$, where $f_{\Omega}$ is a fluxcorrection factor for the fact that the actual phase-averaged flux of a pulsar, integrated over the whole celestial sphere, may be higher $\left(f_{\Omega}>1\right)$ or lower $\left(f_{\Omega}<1\right)$ than would be inferred from assuming isotropic emission based on the phase-averaged flux for the Earth line of sight (Watters et al. 2009).

The phase-averaged flux integrated over the whole sky is a measure of the size of the emission region in the pulsar magnetosphere. In general, values of $f_{\Omega}$ greater than 1 are consistent with the "fan-beam" emission from outer-magnetospheric gaps, whereas PC models tend to produce narrow beams, i.e., $f_{\Omega} \ll 1$.

\footnotetext{
59 http://fermi.gsfc.nasa.gov/ssc/data/analysis/scitools/overview.html
}

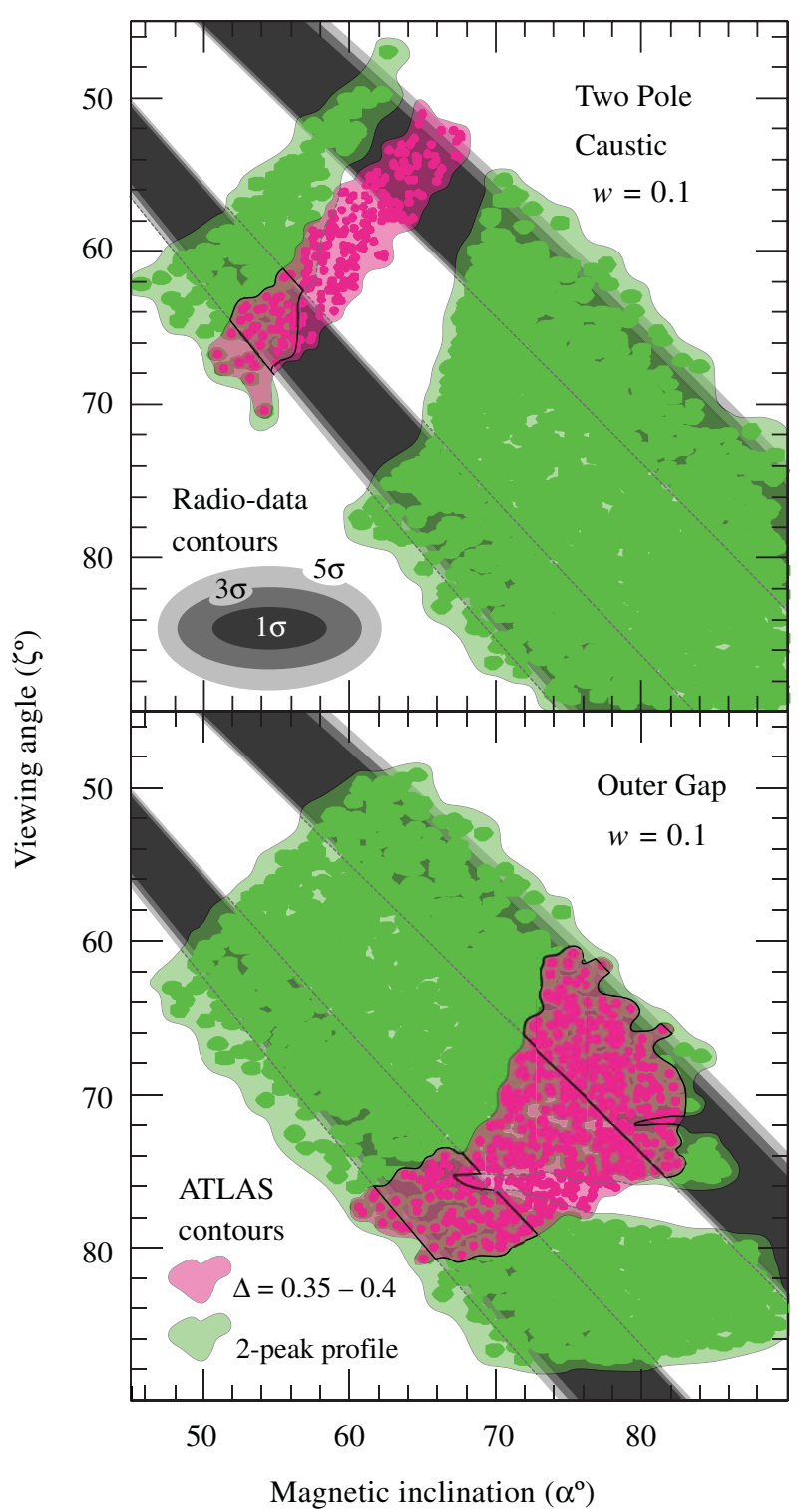

Figure 4. Plots of the magnetic inclination, $\alpha$, vs. the viewing angle, $\zeta$, for $\gamma$ ray pulsars with two-pole caustic emission regions (top) and outer gap regions (bottom), and with an assumed gap thickness of the emission region of $w \sim 0.1$. In these plots, the allowed geometries from the ATLAS maps of Watters et al (2009) are shown with green and pink contours: the green contours correspond to a $\gamma$-ray profile with two major peaks; the pink contours, to a profile with phase separation of $0.35-0.4$ pulse periods between the major peaks. In both models, the geometry is further constrained by the gray-scale contours that were derived from RVM fits to the radio-polarization data of PSR J2043 + 2740. The overlapping regions between the ATLAS and radio contours are delineated with solid, black lines.

(A color version of this figure is available in the online journal.)

The geometrical constraints from our radio-polarization measurements can be combined with information derived from the observed $\gamma$-ray light curve, to place more stringent limits on the allowed values of $\alpha$ and $\zeta$. More specifically, we can use the measured $\mathrm{P} 1-\mathrm{P} 2$ peak separation of $\Delta \approx 0.35$ and the gap-thickness value for PSR J2043 + 2740, $w=\left(L_{\mathrm{sd}} / 10^{33} \mathrm{erg} \mathrm{s}^{-1}\right)^{-1 / 2} \sim 0.1$, defined in Watters et al. (2009), to explore the allowed geometries that are consistent with the TPC and the OG model, based on the ATLAS maps of Watters et al. (2009). Figure 4 shows the $\alpha$ and $\zeta$ values allowed by the RVM fit of the P.A. in gray-scale contours. The green and pink points show the allowed geometries from the ATLAS of Watters et al. (2009), for a two-peaked 
gamma-ray profile and for a profile with a phase separation of $0.35-0.4$ between the major peaks, respectively. The regions where all contours (from the radio data and from the $\gamma$-ray models) overlap are delineated with solid, black lines.

For the TPC model, the overlap between the contours covers roughly the ranges $\alpha \sim 52^{\circ}-57^{\circ}$ and $\zeta \sim 61^{\circ}-68^{\circ}$. For the OG model, there are two separate regions of overlap between the radio contours and the ATLAS maps: these are roughly $\alpha \sim$ $62^{\circ}-73^{\circ}$ and $\zeta \sim 74^{\circ}-81^{\circ}$, and $\alpha \sim 72^{\circ}-83^{\circ}$ and $\zeta \sim 60^{\circ}-75^{\circ}$. The presence of overlapping regions in both TPC and OG contour plots does not allow us to exclude either of the two models. However, it is worth noting that the two outer peaks expected in the TPC geometry both have progressive leading edges and sharp trailing edges, whereas the OG model predicts symmetric peaks (see the Appendix of Watters et al. 2009). Therefore, the gamma-ray light curve shape we observe is consistent with the OG model prediction and inconsistent with the TPC.

Furthermore, as was mentioned earlier, the $\mathrm{P} 1 / \mathrm{P} 2$ ratio decreases with energy. There are already several investigations of the energy dependence of P1 and P2 for Fermi pulsars (Abdo et al. 2010d, 2010e, 2010f). In all those cases, a double-peaked profile is observed that reveals a harder spectral index for P2 compared to that of $\mathrm{P} 1$. This indicates, for curvature radiationdominated models, a higher accelerating electric field and/or a smaller radius of field-line curvature in the $\mathrm{P} 2$ emission region; future modeling can use these spectral variations to probe the underlying physics. Interestingly, the highest-energy $\gamma$ ray is found nearer to P1 than to P2, which means that it is either part of the surviving photons from the respective cutoff process operating near the P1 region, or simply a background photon not associated with the pulsar.

\subsubsection{Altitude of Emission}

An interesting feature that emerges from the radiopolarization profile of PSR J2043 + 2740 is the shape of the circularly polarized flux, which seems to change handedness very near the peak of the total flux (it lags the latter by $\approx 0.6 \%$ of the pulse period). Such features in pulsar profiles have been associated with emission from near the magnetic pole, viewing angles almost along the local magnetic field (Rankin 1986). Interestingly, the RVM fit for this pulsar gives a $\phi_{0}$ that lags the $\mathrm{V}$-swing by $\approx 2 \%$ of the pulse period. According to the original relativistic model of Blaskiewicz et al. (1991), for which Dyks (2008) provided recently a simple explanation in terms of the relative acceleration of the corotating pulsar magnetosphere to the observer's reference frame, such a difference between the fiducial phase of an RVM swing and that of the profile is expected if the emission is altitude dependent. In that case, the radio emission would be generated from an altitude that is roughly $2 \%$ the size of the light cylinder $\left(R_{\mathrm{LC}} \sim 4.59 \times 10^{4} \mathrm{~km}\right)$.

On the other hand, one can place limits on the altitude of the $\gamma$ ray emission based on the highest observed photon energy from PSR J2043 + 2740. The most energetic $\gamma$ ray in the pulsar's light curve corresponds to an energy of $\epsilon_{\max }=4.9 \mathrm{GeV}$. In the framework of a standard PC model, Baring (2004) placed a lower limit on the radius of the high-energy emission, based on $\epsilon_{\max }$. The minimum radius (from the neutron star's center), based on the $\gamma$ $B$ absorption of $\gamma$ rays propagating through the magnetosphere, was estimated to be $r \gtrsim\left(\epsilon_{\max } B_{12} / 1.76 \mathrm{GeV}\right)^{2 / 7} P^{-1 / 7} R_{\star}$, where $R_{\star}$ is the neutron star radius. Substituting for $P=0.0961 \mathrm{~s}$ and $B_{12}=B /\left(10^{12} \mathrm{G}\right)=0.354$, the above inequality yields $r \gtrsim 1.39 R_{\star}$, which rules out emission models that produce $\gamma$ rays very near the PCs. Recently, Lee et al. (2010) performed a largely similar alternative analysis of the altitude of gammaray emission, employing a well-known high-energy asymptotic form for the magnetic pair creation rate. They concluded that the minimum emission height can be approximated with $r \gtrsim 0.11 \times\left(\epsilon_{\max } B_{12}\right)^{2 / 5} P^{-1 / 5} R_{\star}$. Substituting the values of PSR J2043 +2740 , we get $r \gtrsim 3.5 R_{\star}$, which again shows that the highest-energy emission from this pulsar is not produced near the PCs.

\section{2. $\gamma$-ray Efficiency}

As was mentioned in the introduction, the high characteristic age of PSR J2043 + 2740 gives us an opportunity to investigate the claims for a correlation between $\gamma$-ray conversion efficiency and pulsar age, over a wider range of characteristic ages. However, it should be cautioned that any conclusions from such a study should take into account the dependency of both efficiency and characteristic age on the spin parameters, i.e., $\eta=L_{\gamma} / L_{\mathrm{sd}} \propto L_{\gamma}\left(P^{3} / \dot{P}\right)$ and $\tau_{\mathrm{c}} \propto P / \dot{P}$. Therefore, other pulsar parameters that depend on the period and/or its time derivatives, for example, the surface polar field $B$ or the field strength at the light cylinder radius, may well also exhibit a degree of correlation with the efficiency.

The $\gamma$-ray luminosity of a pulsar can be written as $L_{\gamma}=$ $4 \pi f_{\Omega} G_{>0.1 \mathrm{GeV}} D^{2}$. Based on the measured $\gamma$-ray luminosities of non-recycled pulsars recently detected with Fermi, Abdo et al. (2010a) calculated the corresponding $\gamma$-ray efficiencies under the assumption of $f_{\Omega}=1$ and using distance estimates based either on the pulsar DM or known kinematic properties and SNR associations. A scatter plot of the $\gamma$-ray efficiency versus the characteristic age of non-recycled $\gamma$-ray pulsars with available distances is shown in Figure 5. In addition, the plot includes an upper limit on $\eta$, denoted with a solid triangle, corresponding to the efficiency of PSR J1836 + 5925. It should be noted that the efficiency of PSR J1836 + 5925 was recently estimated by Abdo et al. (2010d), assuming $\eta \propto 1 / \sqrt{L_{\mathrm{sd}}}$. However, we did not use their estimate in this work, because such an assumption introduces an artificial correlation between the pulsar's spin parameters and the $\gamma$-ray efficiency.

Despite the large error bars on $L_{\gamma}$, Figure 5 reveals a correlation between $\eta$ and $\tau_{\mathrm{c}}$, with older pulsars having on average higher $\gamma$-ray efficiencies compared to younger ones. The Spearman's rank coefficient for our data set is $r_{\mathrm{s}}=0.57_{-0.07}^{+0.06}$, which implies a positive correlation between efficiency and age. The probability of chance correlation given that value of $r_{\mathrm{s}}$ is only $0.11_{-0.09}^{+0.46 \%}$.

In the plot of Figure 5, the data point corresponding to the highest characteristic age-without being an upper limit-is that of PSR J2043 + 2740 and is denoted by a solid black square. It is notable that the efficiency of PSR J2043 + 2740 is more comparable with that of pulsars that are at least 10 times younger, while the next three youngest pulsars after PSR J2043 + 2740, being roughly half as young, have efficiencies that are two to six times higher. For a number of pulsars in Figure 5, we have two distance estimates: from the pulsar DM and the NE2001 model, and from and independent method; the latter is based either on the Doppler shift of the $\mathrm{H}$ I lines of objects associated with the pulsar, combined with a rotation model for the Galaxy, or on trigonometric parallax, or on estimates by other means (Abdo et al. 2010a). For those pulsars, we have assigned two markers connected with a dashed line, each corresponding to the distance estimate from either method. For PSR J2043 + 2740, we can suppose that its association with the Cygnus Loop is true: this 


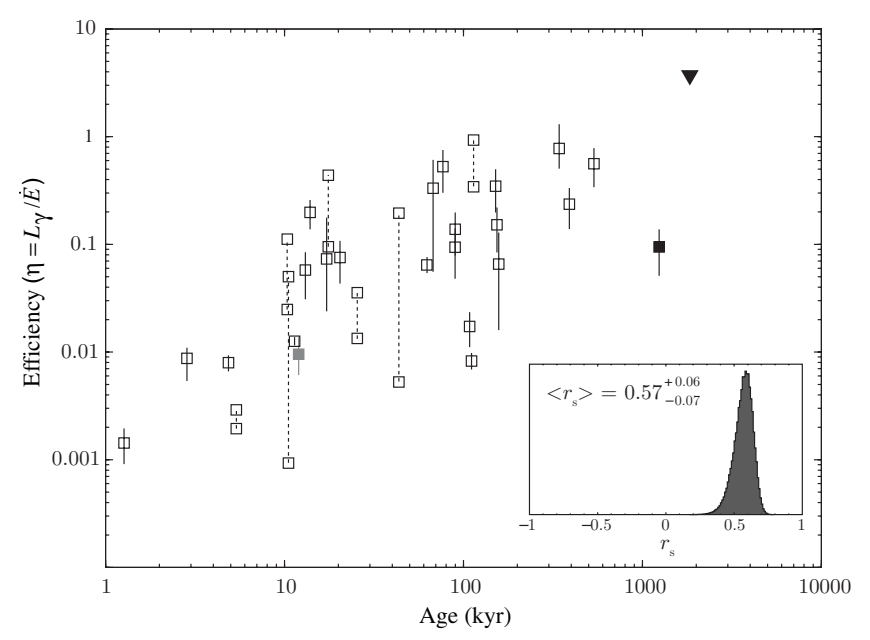

Figure 5. $\gamma$-ray efficiency plotted against characteristic spin-down age for nonrecycled $\gamma$-ray pulsars with available distance estimates (empty squares), and PSR J1836 + 5925, for which only an upper limit exists (solid triangle). Pulsars with two distance estimates have two markers connected with a dashed line. The solid black square corresponds to the efficiency and age of PSR J2043 + 2740; the solid gray square corresponds to the same pulsar's efficiency but assuming its age and distance are equal to those of the Cygnus Loop, i.e., $\tau=12 \mathrm{kyr}$ and $D=$ $540 \mathrm{pc}$. All values of $\eta$ in this plot were taken from Abdo et al. (2010a), except those for PSR J0248 $+6021\left(\tau_{\mathrm{c}}=63 \mathrm{kyr}\right)$ and PSR J2240 $+5832\left(\tau_{\mathrm{c}}=151 \mathrm{kyr}\right)$, which were taken from Theureau et al. (2011). The inset plot, at the bottom right corner, shows the distribution of $10^{6}$ Spearman's rank correlation coefficients, $r_{\mathrm{s}}$, from an equal number of Monte Carlo realisations of the data based on the measured efficiencies and their errors. The median of the distribution is $r_{\mathrm{s}}=0.57_{-0.07}^{+0.06}$, where the uncertainties correspond to the $1 \sigma$ asymmetric errors

in turn would imply that its distance is $\approx 540 \mathrm{pc}$ and, moreover, that its true age is roughly $12 \mathrm{kyr}$ instead of $1.2 \mathrm{Myr}$. Therefore, if the association is true, then the marker for PSR J2043 + 2740 should be shifted to the position of the solid gray square in Figure 5, which would make the efficiency of this pulsar more consistent with the observed overall trend shown by the rest of the data points.

There are indeed a handful of examples for which a pulsar-remnant association has led to a significant revision to the pulsar's age: the case of PSR J0538 + 2817 and S147 has already been mentioned in the introduction; other examples include the 65 ms X-ray pulsar PSR J1811 - 1925, whose association with the Galactic SNR G11.2 - 0.3 suggested an age that was a factor 12 smaller than the spin-down age (Kaspi et al. 2001), and PSR B1951 + 32-one of the original six $\gamma$-ray pulsars detected with EGRET - with a characteristic age that is more than 1.5 times higher than the age of its birth site, the SNR CTB 80 (Migliazzo et al. 2002). The reason for such discrepancies between $\tau_{\mathrm{c}}$ and the true age of a non-recycled pulsar is that the calculation of the characteristic spin-down age assumes in all cases that the pulsar was born with an initial period, $P_{0}$, that is negligible compared to that observed today. Evidently, in the above-mentioned cases, such an assumption does not hold, and those pulsars must have been born with a $P_{0}$ very close to the period observed today. Hence, in the case where $P_{0} \ll P$ does not hold, the true age of the pulsar should be calculated from $\tau=P /[(n-1) \dot{P}] \times\left[1-\left(P_{0} / P\right)^{n-1}\right]$, where $n=2-P \ddot{P} / \dot{P}^{2}$ is the pulsar braking index. If $P_{0} \ll P, \tau$ can be approximated with $\tau_{\mathrm{c}}$. Under the usual assumption of $n=3$, for pure-dipole magnetic braking, one can calculate $\tau$ for a range of $P_{0}$ values. By simply equating the true age of PSR J2043 + 2740 with the upper limit on the age of the Cygnus Loop, one finds that the only possibility of association with the remnant is if the pulsar was born with $P_{0} \approx 95.6 \mathrm{~ms}$.

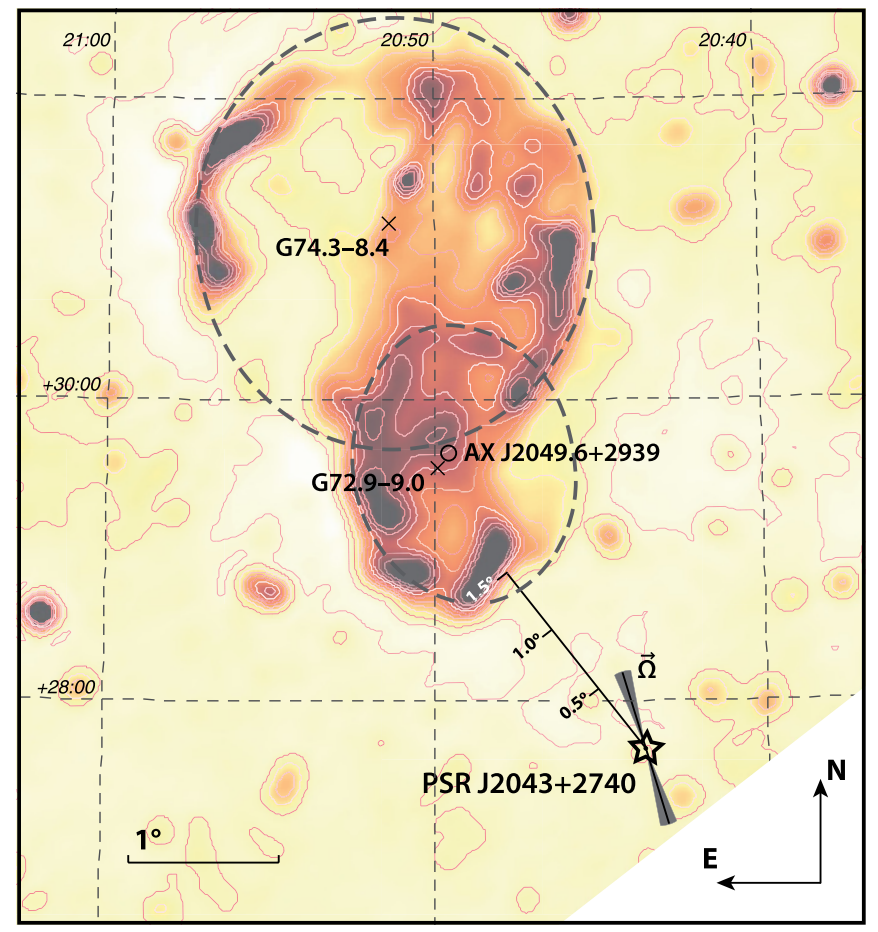

Figure 6. Radio map of the Cygnus Loop region from data from the Effelsberg 1.4 GHz Medium Galactic Latitude Survey (EMLS; Uyanıker et al. 1998,1999; Reich et al. 2004). In this map, the position of PSR J2043 + 2740 is shown with a star symbol. At the pulsar's position, we have also drawn the orientation of the pulsar's spin axis (solid, black line), as was derived from radio-polarization data (the shaded, gray area corresponds to the $1 \sigma$ confidence level). In addition, the centers and extents of G74.3 - 8.4 and G72.9 - 9.0, as were calculated by Uyanıker et al. (2002), are shown with crosses and gray dashed lines, respectively. The position of the X-ray compact source, AX J2049.6 + 2939, discovered by Miyata et al. (1998) is marked with a circle. Lastly, the map also includes a scale showing the angular separation between the pulsar and the edge of the Cygnus Loop.

(A color version of this figure is available in the online journal.)

A conclusive remark based on the above is that there is certainly some support for the pulsar-remnant association on the basis of $\gamma$-ray energetics. Nevertheless, it should be recognized that, as of yet, there exists no firm evidence for such an association and that a chance alignment between PSR J2043 + 2740 and the Cygnus Loop is as likely. Furthermore, it is important to note that a possible rejection of the above association does not provide indisputable support for the alternative distance to PSR J2043 + 2740, i.e., the one derived from the NE2001 model: it is quite possible, given the inaccuracies often associated with distances based on DM (e.g., Deller et al. 2009), that this pulsar is at an even greater distance than predicted by its DM. In fact, PSR J2022 + 2854, which is $\approx 4.5$ away from PSR J2043 + 2740 in the sky, is at a DM distance of $2.7 \mathrm{kpc}$ and has a comparable DM and RM to PSR J2043 + $2740\left(\mathrm{RM}_{\mathrm{J} 2022+2854}=\right.$ $-75 \mathrm{rad} \mathrm{m}^{-2}$ ); while other nearby pulsars, e.g., J2113 + 2754 at $2 \mathrm{kpc}$, have a much lower RM $\left(\mathrm{RM}_{\mathrm{J} 2113+2754}=-37 \mathrm{rad} \mathrm{m}^{-2}\right)$.

\subsection{Pulsar Orientation, Proper Motion, and Birthplace}

The Cygnus Loop region has been searched by a number of authors for the central compact object, but so far there has been no conclusive evidence that led to a positive association (Thorsett et al. 1994; Ray et al. 1996; Miyata et al. 1998, 2001).

Figure 6 shows a radio map of the Cygnus Loop remnant relative to the position of PSR J2043 + 2740; the map was compiled from data from the Effelsberg $1.4 \mathrm{GHz}$ Medium 
Latitude Survey (EMLS; Uyanıker et al. 1998, 1999; Reich et al. 2004). It has been proposed by Uyanıker et al. (2002), and was later supported with polarization observations at $6 \mathrm{~cm}$ (Sun et al. 2006), that the Cygnus Loop consists of two SNRs: the northernmost G74.3 - 8.4, centered at $(\alpha, \delta)=\left(20^{\mathrm{h}} 51^{\mathrm{m}} \cdot 36,+31^{\circ} 3^{\prime}\right)$, and G72.9 - 9.0, a blowout region in the southwestern rim of the Cygnus Loop, centered at $(\alpha, \delta)=\left(20^{\mathrm{h}} 49^{\mathrm{m}} \cdot 56,+29^{\circ} 33^{\prime}\right)$.

Moreover, Miyata et al. (1998) have reported the discovery of a compact X-ray source, AX J2049.6 + 2939, near the center of G72.9-9.0 (see Figure 6). Although it may seem likely that AX J2049.6 + 2939 could be the central compact source associated with G72.9 - 9.0, follow-up observations with the ASCA and $R X T E$ observatories showed significant $\mathrm{X}$-ray variability, which makes the identification of AX J2049.6 + 2939 as an ordinary, rotation-powered pulsar unlikely (Miyata et al. 2001).

Given the lack of alternative candidates, the proximity of PSR J2043 + $2740(\approx 1.5$ outside the edge of G72.9 -9.0$)$ makes this pulsar the only possible prospect for an association. Ultimately, a conclusion to whether PSR J2043 + 2740 is associated with the Cygnus Loop can be drawn by measuring the pulsar's proper motion. One method of estimating pulsar proper motions is through pulsar timing. We examined 7 years of timing observations with the Lovell telescope, at $1.4 \mathrm{GHz}$. We fitted 234 TOAs for the pulsar period and its first and second time derivatives. The post-fit timing residuals displayed the typical, long-term (i.e., "red"), correlated timing signature often seen in the TOAs of non-recycled pulsars: this phenomenon is commonly referred to as "timing noise" (see Hobbs et al. 2004, 2010). Younger and more energetic pulsars often exhibit a higher amount of timing noise than older ones. PSR J2043 + 2740 is ranked amongst the pulsars with the highest amount of timing noise, a fact which is demonstrated by this pulsar's high and significant value of $\ddot{v}=56.04(57) \times 10^{-24} \mathrm{~s}^{-3}$ (compare with Table 1 of Hobbs et al. 2010).

The fitting procedure that can be used to derive the pulsar proper motion from timing data only provides correct results when applied to statistically "white" data. Therefore, some form of pre-whitening needs to be applied to effectively remove the timing noise mentioned above (e.g., Hobbs et al. 2006). We attempted the standard technique of fitting harmonically related sinusoids, but given the strength of the timing noise in this pulsar we were unable to pre-whiten the timing residuals sufficiently to obtain a plausible fit for proper motion.

Another method for inferring the pulsar's proper-motion direction is through our radio-polarization measurements (Section 2.2). It has been claimed that there is strong evidence for a correlation between the projected spin-axis orientations of pulsars and their respective velocity-vector directions (Johnston et al. 2005, 2007). It has also been suggested that such a correlation-if there is indeed a physical mechanism that aligns pulsar spin axes with their velocities - should vanish for old pulsars, as the Galactic gravitational potential will have had enough time to alter the velocities of those pulsars. On the other hand, the young population of pulsars should display a stronger case for alignment.

The position angle P.A. 0 defines the orientation-relative to our polarization dipoles - of the plane of linear polarization of the pulsar's emission, at the closest approach of the magnetic pole to our line of sight. Assuming that the plane of polarization coincides with the plane defined by the magnetic field lines and the magnetic axis, P.A.0 should also correspond to the orientation of the spin axis, projected on our field of view, i.e., P.A.r $\equiv$ P.A.0. Note that the calculation of the P.A. from Stokes $Q$ and $U$ can only provide a "headless" vector with $180^{\circ}$ ambiguity. In addition, as was shown by Backer et al. (1975) and Manchester et al. (1975), pulsar emission can occur in two orthogonal modes, i.e., the polarization plane can either coincide with the field-line-magnetic-axis plane or be perpendicular to it. Therefore, there is an additional $90^{\circ}$ ambiguity in the projected direction of the spin axis.

If we assume that the scenario of alignment between the spin axis and velocity is true for young pulsars and that PSR J2043 + 2740 was indeed born within the bounds of the Cygnus Loop-making it a young pulsar-then we expect that the spin-axis orientation is, within the measurement errors, directed toward the SNR. Our polarization measurements show that the most favorable case to the above scenario, given the $90^{\circ}$ ambiguity, gives a position angle for the spin axis of $17^{\circ}$ north through east. The spin-axis orientation relative to the Cygnus Loop is shown in Figure 6, drawn at the pulsar's position. From the figure, it is immediately evident that the spin-axis orientation is significantly offset from the direction to the center of G72.9 - 9.0, the offset being $\approx 19^{\circ}$.

A direct implication of the above offset could be that our assumptions are not valid and that PSR J2043 + 2740 is not associated with the Cygnus Loop. Another possibility is that PSR J2043 + 2740 was born in the supernova but the velocity vector is not aligned with the pulsar's spin axis: it is true that in the work of Johnston et al. (2005) the offsets between the velocity vectors and the spin axes show a significant spread. However, from the same work, Johnston et al. (2005) derive that roughly $90 \%$ of the pulsars have an offset of $<19^{\circ}$ between the spin-axis orientation and the velocity vector, which makes the measured offset for PSR J2043 + 2740 an unlikely product of any underlying alignment mechanism leading to the observed distribution of offsets.

In conclusion, the above arguments for the pulsar-remnant association, based on the pulsar orientation and its inferred proper-motion direction, do not appear to favor the Cygnus Loop as the birthplace of PSR J2043 + 2740. However, all of these arguments are heavily based on uncertain assumptions; a proper-motion measurement is still required for an unequivocal verdict.

Recently, it was proposed that a large part of the observed timing noise of PSR J2043 + 2740, as well as that of other pulsars, is due to pulse-shape variations (Lyne et al. 2010); those variations appear to be correlated with changes in $\dot{P}$. Moreover, it was suggested that one can fit for such pulse-shape variations and mitigate the large variations in the timing residuals by a significant factor. In particular, PSR J2043 + 2740 shows major pulse variations that cause $100 \%$ change in the pulse's FWHM on time scales of $\approx 200$ days. Our data set, covering nearly 500 days, is certainly affected by those changes; still, for a proper-motion fit that would use years of data-as is needed to obtain a precise measurement-such an effect dominates over the proper-motion signature, i.e., for PSR J2043 + 2740, the rms of the timing residuals over 4000 days is $>1$ s (e.g., see Figure 1 of Lyne et al. 2010). In conclusion, such a method is certain to assist proper-motion estimates through pulsar timing for a large number of "noisy" pulsars, such as PSR J2043 + 2740.

Alternatively, a direct measurement of the pulsar's proper motion can be made with VLBI observations. If the pulsar was born $12 \mathrm{kyr}$ ago in the supernova explosion that created G72.9 - 9.0, then the angular separation between the center of the SNR and the pulsar, i.e., $\approx 2.3$, translates to a transverse 
Table 1

Main Results from the Herein Analysis

\begin{tabular}{|c|c|c|c|}
\hline Property & Value $\left(\right.$ Error $\left.^{\mathrm{a}}\right)$ & Units & Description \\
\hline \multicolumn{4}{|l|}{$\gamma$-ray profile } \\
\hline$\phi_{1}$ & $0.20(3)$ & periods & Phase of leading $\gamma$-ray peak ${ }^{b}$ \\
\hline$\phi_{2}$ & $0.55(2)$ & periods & Phase of trailing $\gamma$-ray peak \\
\hline$w_{1}$ & $0.19(6)$ & periods & Width of leading $\gamma$-ray peak \\
\hline$w_{2}$ & $0.06(1)$ & periods & Width of trailing $\gamma$-ray peak \\
\hline$\Delta$ & $0.35(4)$ & periods & $\gamma$-ray peak separation \\
\hline$\delta$ & $0.20(3)$ & periods & $\gamma$-ray-radio peak separation \\
\hline \multicolumn{4}{|c|}{$\gamma$-ray energetics } \\
\hline$\Gamma$ & $1.1(2)$ & $\cdots$ & Power-law index \\
\hline$E_{\mathrm{c}}$ & $0.8(1)$ & $\mathrm{GeV}$ & Exponential cutoff energy \\
\hline$F_{>0.1 \mathrm{GeV}}$ & $2.2(4)$ & $10^{-8} \mathrm{ph} \mathrm{cm}^{-2} \mathrm{~s}^{-1}$ & Integral photon flux $>0.1 \mathrm{GeV}$ \\
\hline$G_{>0.1 \mathrm{GeV}}$ & $1.5(2)$ & $10^{-11} \mathrm{erg} \mathrm{cm}^{-2} \mathrm{~s}^{-1}$ & Energy flux $>0.1 \mathrm{GeV}$ \\
\hline$L_{\gamma}$ & $5.3(24)^{\mathrm{c}}$ & $10^{33} \mathrm{erg} \mathrm{s}^{-1}$ & $\gamma$-ray luminosity $>0.1 \mathrm{GeV}$ \\
\hline$\eta_{\gamma}$ & $0.09(4)$ & $10^{-11} \mathrm{erg} \mathrm{cm}^{-2} \mathrm{~s}^{-1}$ & $\gamma$-ray efficiency $>0.1 \mathrm{GeV}$ \\
\hline \multicolumn{4}{|c|}{ Geometry and polarization } \\
\hline$\alpha(\mathrm{TPC} / \mathrm{OG})$ & \multirow{2}{*}{\multicolumn{2}{|c|}{$\begin{array}{r}52^{\circ}-57^{\circ} / 62^{\circ}-73^{\circ} \text { or } 72^{\circ}-83^{\circ} \\
61^{\circ}-68^{\circ} / 74^{\circ}-81^{\circ} \text { or } 60^{\circ}-75^{\circ}\end{array}$}} & Inclination angle ranges ${ }^{\mathrm{d}}$ \\
\hline$\zeta(\mathrm{TPC} / \mathrm{OG})$ & & & Viewing angle ranges \\
\hline $\mathrm{RM}$ & $-92.7(19)$ & \multirow{3}{*}{$\begin{array}{l}\text { rad m}^{-2} \\
\text { periods } \\
\text { degrees }\end{array}$} & Rotation measure \\
\hline$\phi_{0}$ & $0.032(2)$ & & nimum approach to the magnetic pole $\mathrm{e}^{\mathrm{e}}$ \\
\hline P.A.r & $17(6)$ & & Spin-axis position angle $\mathrm{f}^{\mathrm{f}}$ \\
\hline
\end{tabular}

Notes.

a All errors in parentheses are on the last significant digit of the value to which they refer.

b Relative to the radio peak.

c The error on $L_{\gamma}$ incorporates the uncertainties in $G_{>0.1 \mathrm{GeV}}$ and $D$. All flux calculations assume $f_{\Omega}=1$.

${ }^{\mathrm{d}}$ Each range of $\alpha$ is matched with the corresponding range of $\zeta$, directly beneath it.

e Relative to the maximum of the radio emission; positive values denote delay.

${ }^{\mathrm{f}}$ Measured north through east.

velocity of $V_{\perp} \sim 1770 \mathrm{~km} \mathrm{~s}^{-1}$, assuming both pulsar and remnant are at $540 \mathrm{pc}$ distance. Indeed, this corresponds to a large value of proper motion $\left(\approx 690\right.$ mas $\left.\mathrm{yr}^{-1}\right)$, with the highest inferred transverse velocities published so far being $V_{\perp} \sim 1600 \mathrm{~km} \mathrm{~s}^{-1}$ (Hobbs et al. 2005). We have performed TEMPO2 simulations that generate fake TOAs having a propermotion signature corresponding to a pulsar at the position of PSR J2043 + 2740, with 690 mas $\mathrm{yr}^{-1}$. Fitting those TOAs with the pulsar's spin parameters but setting the proper motion to zero reveals the characteristic periodic sine wave, modulated with time difference from the reference position epoch. The maximum amplitude of the simulated wave over a few thousand days, due to the proper motion, was $\sim 10 \mathrm{~ms}$ (see also Figure 8.2(d) in Lorimer \& Kramer 2005). This otherwise large amount of residual rms is still swamped, for the case of PSR J2043 + 2740, by the much larger timing noise of this pulsar. However, if PSR J2043 + 2740 possesses such a large proper motion, it will be easily measurable with VLBI, which will conclusively confirm or reject the pulsar-remnant association.

\section{SUMMARY}

The present article presented the results of the analysis of radio and $\gamma$-ray data from PSR J2043 +2740 , taken with the Jodrell Bank and Effelsberg radio telescopes, and the Fermi $\gamma$ ray observatory. Table 1 gives a summary of the main numerical results from the above analysis.

We used a radio ephemeris from Jodrell Bank timing observations to fold 14 months of Fermi $\gamma$-ray data from PSR J2043 +2740 . The resulting $\gamma$-ray light curve clearly shows a double-peaked structure with a significance of $\approx 7 \sigma$.
Using a bin-independent statistical analysis, we derived a pulse width of $\approx 0.19$ pulse periods for the leading $\gamma$-ray peak and a significantly narrower width of $\approx 0.06$ pulse periods for the trailing peak. The highest-energy event present in the constructed light curve has $E=4.9 \mathrm{GeV}$ and was found coincident with the bridge emission between the $\gamma$-ray peaks.

The significant detection of a $\gamma$-ray light curve from PSR J2043 + 2740 allowed us to further characterize the properties of the pulsar's $\gamma$-ray emission: we have measured a phase lag of $\delta \approx 0.2$ pulse periods between the radio and $\gamma$-ray emission; we also measured the phase separation between the major $\gamma$-ray peaks to be $\Delta \approx 0.35$ pulse periods.

The shape and structure of the $\gamma$-ray light curve allowed us to constrain the beam geometry of PSR J2043 + 2740 using the recently published ATLAS of $\gamma$-ray light curves by Watters et al. We only considered the ATLAS results from the two prevailing outer-magnetospheric models: the TPC model and the OG model. Our assumption that the $\gamma$-ray emission for PSR J2043 + 2740 comes from the outer magnetosphere was also supported by theoretical estimates based on the highest energy detectable from this pulsar: we estimated that the highenergy emission most likely comes from a distance of at least $1.4 R_{\star}$ from the star's center.

We combined the geometrical constraints from the $\gamma$-ray data with the results from $21 \mathrm{~cm}$ polarization observations of PSR J2043 + 2740 with the Effelsberg telescope: by fitting the rotating vector model of Radhakrishnan \& Cooke (1969) to the absolute polarization angles across the radio pulse, we produced confidence contours constraining the magnetic inclination and viewing angles. The $\gamma$-ray and radio constraints combined place stringent limits on the allowed ranges for these angles. For 
the TPC model, we determined $\{\alpha, \zeta\} \sim\left\{52^{\circ}-57^{\circ}, 61^{\circ}-68^{\circ}\right\}$ to be a valid parameter range. For the OG model, we found two separate ranges that satisfied the data constraints: $\{\alpha, \zeta\} \sim$ $\left\{62^{\circ}-73^{\circ}, 74^{\circ}-81^{\circ}\right\}$ and $\{\alpha, \zeta\} \sim\left\{72^{\circ}-83^{\circ}, 60^{\circ}-75^{\circ}\right\}$.

Given the presence of valid ranges for $\alpha$ and $\zeta$ for both models, we could not exclude either of them based on emissiongeometry arguments. However, it was noted that the roughly symmetric shapes of the $\gamma$-ray peaks in the light curve of PSR J2043 +2740 favor the predictions of the OG model but are incompatible with the asymmetric peaks predicted by the TPC model.

In addition, we performed a spectral analysis on all $\gamma$-ray events above $0.1 \mathrm{GeV}$, from the direction of PSR J2043 + 2740 . The best fit to the measured fluxes as a function of photon energy was compatible with a power law of spectral index $1.1 \pm 0.2$ and an exponential cutoff at $0.8 \pm 0.1 \mathrm{GeV}$.

Previous claims that pulsars become more efficient at emitting $\gamma$ rays as they age were also investigated in this paper. We plotted the $\gamma$-ray efficiency of PSR J2043 +2740 together with those of other non-recycled $\gamma$-ray pulsars as a function of characteristic age. Despite the large scatter present in the data, we saw an evident trend toward higher efficiencies with growing pulsar age. The position of PSR J2043 +2740 in our plot is somewhat lower than the expected efficiency based on the average trend of the total sample.

Furthermore, we have also explored the possibility that PSR J2043 + 2740 was born in the nearby, 12 kyr SNR, the Cygnus Loop. By using the pulsar-remnant association as a postulate, we concluded that the pulsar's $\gamma$-ray efficiency is comparable, within the measurement errors, to that of most other pulsars of similar characteristic age as the age of the Cygnus Loop.

In an attempt to make a conclusive statement about the above association, we used Jodrell Bank timing data from PSR J2043 + 2740, in order to measure its proper motion through pulsar timing: a proper-motion vector pointing away from the center of the Cygnus Loop would unquestionably make the latter the pulsar's birthplace. Unfortunately, due to the high timing noise present in the pulsar's timing residuals, we were unable to fit for proper motion.

Alternatively, we considered an indirect method of estimating the pulsar's proper-motion direction, based on the claims of Johnston et al. $(2005,2007)$ that young pulsars tend to have their spin axes and proper-motion vectors aligned. Therefore, if PSR J2043 + 2740 is associated with the Cygnus Loop, it should be young and have a proper motion directed along the spin axis. From the rotating-vector-model fits to the radiopolarization angles, the best determined value of the spin-axis orientation of PSR J2043 +2740 was $17^{\circ} \pm 6^{\circ}$, measured from north through east. The derived spin-axis direction points $19^{\circ}$ away from the nearest SNR center, i.e., that of the Cygnus Loop's southwestern blowout. This result weakens the arguments for an association: it means that either PSR J2043 + 2740 was not born in the Cygnus Loop or possibly_although unlikely-that the velocity vector of this pulsar is not aligned with its spin axis and that the former does in fact point away from the center of the SNR. If the latter is true, our calculations showed that the current angular separation between PSR J2043 + 2740 and the southwestern part of the Cygnus Loop implies a transverse velocity of $V_{\perp} \sim 1770 \mathrm{~km} \mathrm{~s}^{-1}$ for the pulsar. If this pulsar is indeed moving away from the Cygnus Loop at such a high velocity, future VLBI measurements should be able to easily measure it and shed light on this pulsar's connection to the Cygnus Loop.
The Fermi LAT Collaboration acknowledges generous ongoing support from a number of agencies and institutes that have supported both the development and the operation of the LAT as well as scientific data analysis. These include the National Aeronautics and Space Administration and the Department of Energy in the United States, the Commissariat à l'Energie Atomique and the Centre National de la Recherche Scientifique/Institut National de Physique Nucléaire et de Physique des Particules in France, the Agenzia Spaziale Italiana and the Istituto Nazionale di Fisica Nucleare in Italy, the Ministry of Education,Culture, Sports, Science and Technology (MEXT), High Energy Accelerator Research Organization (KEK) and Japan Aerospace Exploration Agency (JAXA) in Japan, and the K. A. Wallenberg Foundation, the Swedish Research Council, and the Swedish National Space Board in Sweden.

Additional support for science analysis during the operations phase is gratefully acknowledged from the Istituto Nazionale di Astrofisica in Italy and the Centre National d'Études Spatiales in France.

The Lovell Telescope is owned and operated by the University of Manchester as part of the Jodrell Bank Centre for Astrophysics with support from the Science and Technology Facilities Council of the United Kingdom.

The authors give special thanks to Drs. K. J. Lee, W. Reich, and J. Verbiest for their invaluable contribution and helpful comments, which helped to vastly improve the present article.

\section{REFERENCES}

Abdo, A. A., et al. 2009, ApJ, 696, 1084

Abdo, A. A., et al. 2010a, ApJ, 713, 154

Abdo, A. A., et al. 2010b, ApJS, 187, 460

Abdo, A. A., et al. 2010c, ApJ, 708, 1254

Abdo, A. A., et al. 2010d, ApJ, 712, 1209

Abdo, A. A., et al. 2010e, ApJ, 720, 272

Abdo, A. A., et al. 2010f, ApJ, 720, 26

Anderson, S., Cadwell, B. J., Jacoby, B. A., Wolszczan, A., Foster, R. S., \& Kramer, M. 1996, ApJ, 468, L55

Atwood, W. B., et al. 2009, ApJ, 697, 1071

Backer, D. C., Rankin, J. M., \& Campbell, D. B. 1975, ApJ, 197, 481

Baring, M. G. 2004, Adv. Space Res., 33, 552

Blair, W. P., Sankrit, R., \& Raymond, J. C. 2005, AJ, 129, 2268

Blair, W. P., Sankrit, R., Torres, S. I., Chayer, P., \& Danforth, C. W. 2009, ApJ, 692, 335

Blaskiewicz, M., Cordes, J. M., \& Wasserman, I. 1991, ApJ, 370, 643

Buccheri, R. 1980, in Non-Solar Gamma-Rays, ed. R. Cowsik \& R. D. Wills (Oxford: Pergamon), 17

Carr, P. 2007, PhD thesis, Univ. Manchester

Cheng, K. S., \& Zhang, L. 1998, ApJ, 498, 327

Cordes, J. M., \& Lazio, T. J. W. 2002, arXiv:astro-ph/0207156

de Jager, O. C. 1994, ApJ, 436, 239

de Jager, O. C., \& Büsching, I. 2010, A\&A, 517, L9

de Jager, O. C., Swanepoel, J. W. H., \& Raubenheimer, B. C. 1989, A\&A, 221, 180

Deller, A. T., Tingay, S. J., Bailes, M., \& Reynolds, J. E. 2009, ApJ, 701, 1243 Dyks, J. 2008, MNRAS, 391, 859

Dyks, J., \& Rudak, B. 2003, ApJ, 598, 1201

Harding, A. K. 1981, ApJ, 245, 267

Hartman, R. C., et al. 1999, ApJS, 123, 79

Hibschman, J. A. 2002, ApJ, 565, 1183

Hobbs, G. B., Edwards, R. T., \& Manchester, R. N. 2006, MNRAS, 369, 655

Hobbs, G., Lorimer, D. R., Lyne, A. G., \& Kramer, M. 2005, MNRAS, 360, 974

Hobbs, G., Lyne, A. G., \& Kramer, M. 2010, MNRAS, 402, 1027

Hobbs, G., Lyne, A. G., Kramer, M., Martin, C. E., \& Jordan, C. 2004, MNRAS, 353,1311

Hotan, A. W., van Straten, W., \& Manchester, R. N. 2004, PASA, 21, 302

Johnston, S. 2002, PASA, 19, 277

Johnston, S., Hobbs, G., Vigeland, S., Kramer, M., Weisberg, J. M., \& Lyne, A. G. 2005, MNRAS, 364, 1397 
Johnston, S., Kramer, M., Karastergiou, A., Hobbs, G., Ord, S., \& Wallman, J. 2007, MNRAS, 381, 1625

Kaspi, V. M., Roberts, M. S. E., Vasisht, G., Gotthelf, E. V., Pivovaroff, M., \& Kawai, N. 2001, ApJ, 560, 371

Kramer, M., Lyne, A. G., Hobbs, G., Löhmer, O., Carr, P., Jordan, C., \& Wolszczan, A. 2003, ApJ, 593, L31

Lee, K. J., Du, Y. J., Wang, H. G., Qiao, G. J., Xu, R. X., \& Han, J. L. 2010 , MNRAS, 405, 2103

Lorimer, D. R., \& Kramer, M. 2005, Handbook of Pulsar Astronomy (Cambridge: Cambridge Univ. Press)

Lyne, A., Hobbs, G., Kramer, M., Stairs, I., \& Stappers, B. 2010, Science, 329, 408

Manchester, R. N., Hobbs, G. B., Teoh, A., \& Hobbs, M. 2005, AJ, 129, 1993

Manchester, R. N., Taylor, J. H., \& Huguenin, G. R. 1975, ApJ, 196, 83

Mattox, J. R., et al. 1996, ApJ, 461, 396

McGowan, K. E., Kenea, J. A., Zane, S., Cordova, F. A., Cropper, M., Ho, C., Sasseen, T., \& Vestrand, W. T. 2003, ApJ, 591, 380

McLaughlin, M. A., \& Cordes, J. M. 2000, ApJ, 538, 818

Migliazzo, J. M., Gaensler, B. M., Backer, D. C., Stappers, B. W., van der Swaluw, E., \& Strom, R. G. 2002, ApJ, 567, L141

Miyata, E., Ohta, K., Torii, K., Takeshima, T., Tsunemi, H., Hasegawa, T., \& Hashimoto, Y. 2001, ApJ, 550, 1023

Miyata, E., et al. 1998, PASJ, 50, 475

Ng, C.-Y., Romani, R. W., Brisken, W. F., Chatterjee, S., \& Kramer, M. 2007, ApJ, 654, 487

Pellizzoni, A., et al. 2009, ApJ, 695, L115
Radhakrishnan, V., \& Cooke, D. J. 1969, Astrophys. Lett., 3, 225

Rankin, J. M. 1986, ApJ, 301, 901

Ray, P. S., Thorsett, S. E., Jenet, F. A., van Kerkwijk, M. H., Kulkarni, S. R., Prince, T. A., Sandhu, J. S., \& Nice, D. J. 1996, ApJ, 470, 1103

Reich, W., Fürst, E., Reich, P., Uyaniker, B., Wielebinski, R., \& Wolleben, M. 2004, in The Magnetized Interstellar Medium, ed. B. Uyaniker, W. Reich, \& R. Wielebinski (Katlenburg-Lindau: Copernicus GmbH), 45

Romani, R. W., \& Ng, C.-Y. 2003, ApJ, 585, L41

Rudak, B., \& Dyks, J. 1998, MNRAS, 295, 337

Sankrit, R., \& Blair, W. P. 2002, ApJ, 565, 297

Smith, D. A., et al. 2008, A\&A, 492, 923

Sun, X. H., Reich, W., Han, J. L., Reich, P., \& Wielebinski, R. 2006, A\&A, 447, 937

Theureau, G., et al. 2011, A\&A, 525, A94

Thompson, D. J., et al. 1999, ApJ, 516, 297

Thorsett, S. E., Ray, P. S., Kulkarni, S. R., \& Prince, T. A. 1994, IAU Circ., 6012

Uyanıker, B., Fürst, E., Reich, W., Reich, P., \& Wielebinski, R. 1998, A\&AS, 132,401

Uyanıker, B., Fürst, E., Reich, W., Reich, P., \& Wielebinski, R. 1999, A\&AS, 138,31

Uyanıker, B., Reich, W., Yar, A., Kothes, R., \& Fürst, E. 2002, A\&A, 389, L61

Watters, K. P., Romani, R. W., Weltevrede, P., \& Johnston, S. 2009, ApJ, 695 , 1289

Zhang, L., \& Cheng, K. S. 1998, A\&A, 335, 234 